\title{
Strong Constraint Dynamic Simulation Method for 3D Garment Models
}

\author{
Zheng-Dong Liu \\ Computer Information Center, Beijing Institute of Fashion Technology, Beijing, P. R. China \\ E-mail: jsjlzd@bift.edu.cn
}

\begin{abstract}
With the continuous improvement of quality of 3D games and virtual fitting, the trend of garment simulation has changed: from cloth simulation to dynamic simulation. Garment simulation is of significant theoretical and practical value, because it is authentic, real-timely, universal and interactive. To solve the problems commonly existing in present algorisms on timeliness, universality and overstretching, a real-timely simulation method for all 3D garment models was proposed. In this method, the triangle faces were mapped to the mass-spring model, which overcame the restriction effect of the traditional solver on cloth regular grid. The Verlet integrator easy to improve the computation efficiency. We put compensation on confrontation of deformation constraint, which prevented over-stretching and improved the stability of the system. The efficiency and reliability of the proposed algorithm was verified by experiments. The experiment results show that this algorithm is simple and easy to operate, and that it can be easily integrated, in the form of plug-in components and modules, with other software.
\end{abstract}

Keywords-garment simulation; mass-spring model; triangle faces; verlet

\section{INTRODUCTION}

Cloth simulation has been a hotspot and a difficulty in the research for many years. As a flexible object, cloth with the surface shape varying at any moment is different from the common rigid body, whose surface deformation can almost be negligible. Then there will be more problems to be solved in the simulation of $3 \mathrm{D}$ garment consisting of cloth and garment pieces. In addition to the real-time performance, authenticity and interactivity are also required in the complex cloth-body collision detection. Actually no matter if it's on an international scale or in China, researches on flexible cloth and 3D garment modeling and simulation have aroused wide attention, promising a broad application prospect specifically described as below in different fields in addition to the theoretical and technical challenges:

- Garment aided design and production. Capable to realize design visualization, virtual 3D cutting, the automatic formation of garment pieces and garment pattern making.

- 3D animation and games. Capable to achieve realtime simulation of the role's dressing effect to give the players a more authentic experience.

- Marketing requirement of E-commerce. With the development of "Internet+" and Garment O2O, it's inevitable that virtual trying on of garment products will become a link since the static model show has been far from meeting the increasing requirements of the consumers.

- Individualized garment customization. Individualized customization has become an important trend for the development of garment design and making. Same with the requirement of Ecommerce, on-line experience is also necessary to the consumers, whose consuming intention will be affected directly by the virtual dressing effect.

Garment simulation starts from the simulation of a single piece. On account of the elastic property of woven cloth, Provot [1] has considered the cloth object as a grid structure constituted by virtual mass and spring to calculate the stress and movement condition of each mass by solving the Euler equations through the iterative solution method. Baraff et al. [2] has proposed an equation of motion for cloth simulation with the application of implicit large-step integration algorithm to strengthen in real-time the constraints on particles. In this way, the computing speed will be improved significantly. Although not all of the cloth motion equations have been expressed in the same way by the researchers, most of them are developed in the physical model based on Newtonian motion [3-7]. On account of the over-stretching in cloth deformation, Vassilev et al. [4, 5, and 14] have set an elongation threshold in addition to the introduction of balancing forces. When the stretch of a spring is detected exceeding the elongation threshold, increase the spring force to restore the spring to a proper position. However, it might lead to spring vibration, which would affect the system stability. In view of the bottlenecks, such as real-time performance and complexity in the "cloth-body" collision detection, Mao Tianlu et al. [8] have proposed a solution to reduce the complexity in collision detection time without the need to update massive intermediate data. In order to obtain the mapping relation between the fabric mechanical properties and the parameters of the simulation model, Jiang Yan et al. [9] have made an attempt to make the simulated fabric deformation compliant with the physical property of the real fabrics. In order to achieve the perception by two fingers and for the purpose to display the fabric deformation effect, Huang Xin et al. [10] have tried an interactive multicontact prototype system for virtual fabrics. As to the research on the garment simulation in the field of garmentoriented aided design, generally it focuses more on how to simulate the real dressing effect [11] with the designed garment pieces according to the virtual reality technology. Generally, a mapping model from 2D garment pieces to 3D garment will be established to provide the composition of the internal force and the external force imposed on the mass by calculating the realistic garment simulation equation in 
addition to the display of the garment draping effect [12-14]. Or in reverse, firstly generate a 3D garment surface and then unfold it into 2D garment pieces for industrial production through the interactive design. Furthermore, due to the availability of the interactive somatosensory devices in recent years, some commercial 2D or 3D dressing systems have also come along, where somatosensory information from the sensor has been used to process separately the position and posture of the human body and garment. However, it's not a true dynamic garment try-on, since the dynamic and physical properties of the garment have been eschewed in such a system.

Although various improvement methods have continuously pushed forward the enhancement of the flexible cloth simulation level, most of them still focus on improving the efficiency of the local algorithms without taking the application scope and the efficiency of the algorithms into account from an overall perspective. Currently garment simulation technology is yet to be improved in the following aspects:

- Timeliness. It's quite time-consuming to make the motion trail of a substantial amount of mass points contained in the cloth conform to the characteristics of real fabrics. In the iterative process, it's necessary to calculate repeatedly the equation of particle dynamics since collision detection and response has also constituted one of the major factors that influence the computing efficiency. Hence through the commercial cloth solver, lots of time will be spent on the rendering instead of real-time simulation.

- Universality. Through the early methods, only rectangular plane will be utilized to simulate cloth. Also in current researches, most of them still adopt customized garment models, such as T-shirt and skirt etc. that have been created through the regular gridding method. Even in some of the models, 3D garment is generated by joining the 2D garment pieces. However nowadays lots of ordinary garment models have been created with the 3D modeling software, containing numerous irregular triangle faces to make the existing methods seem not so practical and flexible. Sometimes artificial process is necessary in such models.

- Efficient "cloth-body" collision detection. Due to the complexity of human body surface and the flexible characteristics of fabric surface, lots of time will be spent on the collision detection and response. Meanwhile the error matrix-based simplified algorithm for grid faces in itself is rather timeconsuming [15]. Also it's yet to be improved regarding the triangle intersection calculation and the inner point recognition.

Aiming at the problem of timeliness and universality, this paper proposes a simulation method for 3D garment model with an aim to make such a method applicable to any garment model that has been created based on the universal modeling software to break through the limitation on the regular grid model, which might then be a collection of irregular triangle faces. In this case, map the triangle vertexes into a link list according to the adjacency relation to construct a mass-spring structure for a random 3D model. Then use the efficient Verlet integrator to make a rapid prediction about the coordinates for mass motion with the application of strong constraint on the rigid spring to correct the predicted position so as to prevent the occurrence of over-stretching. As to the cloth-body collision detection problem, it will be discussed separately in another paper.

\section{VERLET-BASED MASS MOTION MODEL}

Typical mass-spring model is actually a grid that consists of $m \times n$ virtual mass points, which are connected with a mass-less spring, whose natural length is non-zero. Springs can be classified into three different types according to the mechanical force in the cloth: the structural spring, the shearing spring and the flexural spring, which correspond separately to the calculation of the structural force, the shearing force and the bending force in the cloth. The massspring structure is shown in Figure 1.

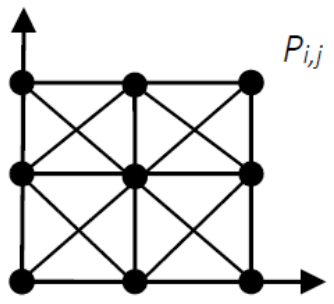

(a) Mass-spring model

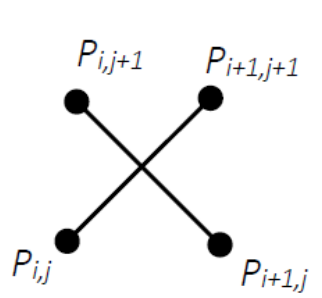

(c) Shearing spring

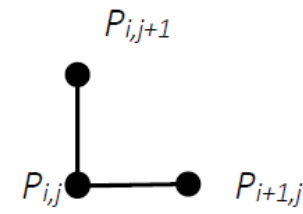

(b) Structural spring

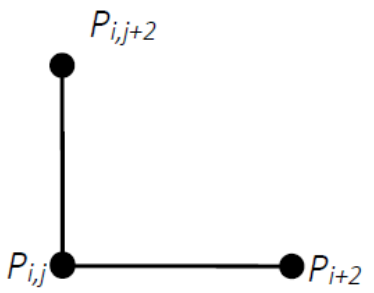

(d) Flexural spring
Figure 1. Mass-spring model and the three types of springs.

In Figure 1, the spring that connects the closely linked horizontal and longitudinal mass points is the structural spring, which is used to secure the fabric structure. The spring that connects the adjacent mass points on a diagonal line is the shearing spring, which is used to prevent the cloth from artificial transitional deformation on the surface, bringing shear rigidity to the cloth. The spring that connects a horizontal and a longitudinal mass point, which are separated by a mass point is the flexural spring, which is used to simulate the flexural loading on the cloth.

The core of simulation is the mass motion system. Although multiple algorithms are available to obtain the 
numerical solution, there's a common starting point, which is to expand the particle position and the other mechanics parameters (such as speed and acceleration etc.) into a Taylor series. Actually the most common numerical solution method is the Verlet algorithm [15, 16], which utilizes the particle position $p(t)$ at $t$ moment, the acceleration $a(t)$ and the particle position at the moment of $t-\Delta t$ to calculate the particle position at the moment of $t+\delta t$.

$$
p(t+\Delta t)=2 p(t)-p(t-\Delta t)+\frac{d^{2}}{d t^{2}} p(t)(\Delta t)^{2}
$$

In (1), no explicit calculation is made on the particle velocity. Since $\frac{d^{2}}{d t^{2}} p(t)=a(t)$, it's applicable to make a direct prediction about the particle position at the moment of $t+\Delta t$ according to its positions at $t$ moment and at the moment of $t-\Delta t$. At the end of every time step, the previous position and the current position of every mass point will be reserved. Then in the processing of a highprecision garment model, the simple exchange of array pointers will facilitate the enhancement of the computation efficiency.

Regarding the forces imposed on the various mass points in the cloth, use Newton's laws of motion $f=$ ma to calculate the current acceleration with $f$ referring to the resultant force imposed on the mass. Taking the typical simulation of cloth falling as an instance, Figure 2 shows the simulation effect through the Verlet algorithm.
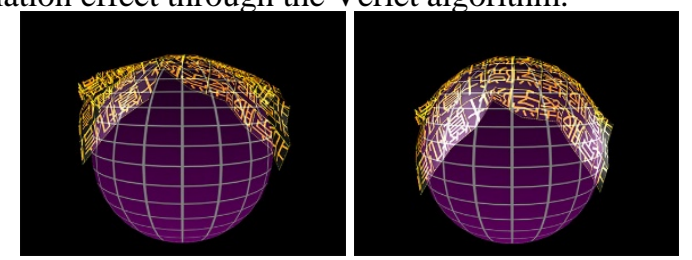

Figure 2. Simulation of cloth falling under the effect of gravity.

In the whole simulation process, only three position coordinates, including the coordinates of the previous position, the current position and the predicted position for every vertex are calculated. $|p(t)-p(t-\Delta t)|$, the difference between the current position and the previous position represents the convergence of each iterative process. Taking one of the vertexes as an instance, Figure 3 shows its iterative convergence curve.

\section{STRONG CONSTRAINT FOR OVER- STRETCHING}

In garment simulation, if an ideal mass-spring model has been adopted to simulate the forced deformation of the cloth, there will be a proportional relation between the spring elongation and the stress on the spring. However, the deformation result through such a simulation method would look false since "over-stretching", which can also be called as "super stretch" would occur in the iterative process. Figure 4 shows the different states of a 9x9 regular grid cloth, which is fixed at the top left corner and the top right corner with different levels of stretch under the action of gravity. The left image shows the state of cloth when the overstretching is within $5 \%$. And the right image shows the state of cloth when the over-stretching is up to $20 \%$. Both of the images reveal that the simulation effect is quite similar to the real state when the over-stretching is controlled within $5 \%$. In fact, required by the real-time 3D game and the dressing system, there shouldn't be apparent cloth stretch under the effect of gravity. However, for performance enhancement purpose, nowadays lots of algorithms have deliberately ignored such a fact.

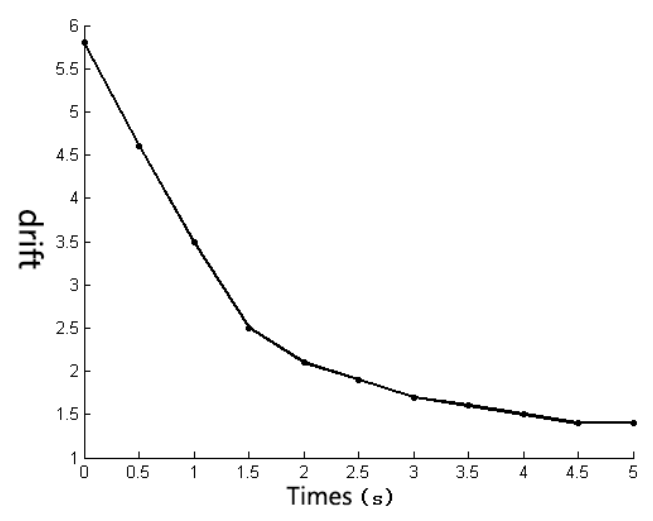

Figure 3. Iterative convergence of a single mass point

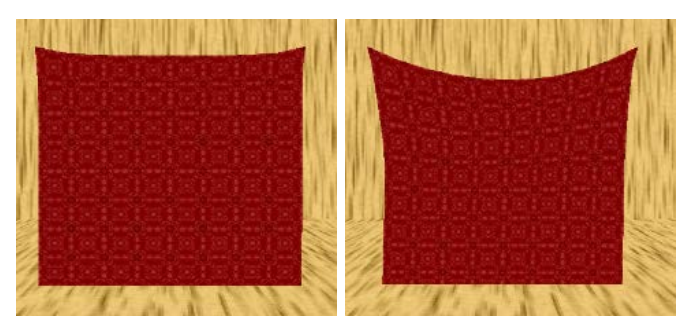

Figure 4. “Over-stretching” of the cloth.

In order to achieve an authentic simulation effect in garment simulation and to avoid the occurrence of overstretching (super stretch), it's necessary to make a timely correction on the positions of various mass points in the iterative process. Through the traditional practices $[1,5,18]$, first calculate the positions of various mass points in an ideal spring model and then calculate the stretch rate of different springs to impose a constraint on the mass points at both sides of the spring when and only when the spring elongation exceeds the preset elongation threshold. That's to say, this method allows spring over-stretching with an attempt made on the adjustment of the spring length, when the spring is stretched. However, since the springs are connected through the mass points, then the change of a spring position might lead to the over-stretching of the spring that is adjacent to it, bringing about the fluctuation in the position to affect the convergence of the algorithm. On account of this, new coordinate can be called as predicted coordinate, since it might be an error position, where over-stretching might occur. Under such a circumstance, it's not applicable to determine the coordinate before the imposition of constraint conditions. The specific approach is provided as below: 
Check if the state of every spring is compliant with the constraint condition after each iteration process. If not, make a compensatory correction on the predicted coordinate so that the corrected coordinate can be used as the iteration result.

Assume that the mass points at both ends of a spring within the mass-spring model are separately $V_{i}$ and $V_{j}$, then its initial spring can be expressed with $L_{i j}$, which can also be used as the bilateral constraints:

$$
L_{i j}=p_{i}-p_{j}
$$

Although in the initial state, the particle positions are correct, the distance between the points might still become null after several time steps of iteration. Hence in order to obtain the correct distance between the mass points once again, it's necessary to make an adjustment through the displacement compensation according to the constraint condition by directly pushing away or pulling back the mass points along the direction of the spring. However, whether to push away or pull back the mass points is subject to the error distance.

In the case that $\dot{L}_{i j}^{\prime}=p_{i}^{\prime}-p_{j}^{\prime}$ is defined as the new vector of the predicted spring, the compensation vector for the coordinate of the mass point can be expressed with (3):

$$
\Delta p_{i}=-\frac{\dot{L}_{i j}\left(\left|\dot{L}_{i j}\right|-\left|L_{i j}\right|\right)}{2\left|\dot{L}_{i j}\right|} \Delta p_{j}=\frac{\dot{L}_{i j}\left(\left|\dot{L}_{i j}\right|-\left|L_{i j}\right|\right)}{2\left|\dot{L}_{i j}\right|}
$$

In conclusion, the following major links are necessary in the simulation of any garment model consisting of triangle faces, including the mapping of the mass-spring structure, the prediction on the coordinates of the mass points and the constraint compensation etc. A stable coordinate will be gradually obtained after the iterative process with the iteration flow described as below:

Input: 3D garment model

- Step 1: Read in the data about the vertexes and the triangle faces of the model and then calculate the structural spring and the flexural spring between the sets of particles according to the mapping relation to initialize $p(t+\Delta t)=p(t)=p(t-\Delta t)$.

- Step 2: Impose such external forces as gravity, wind power and artificial force etc. on the mass points. Turn to Step 4 if the resultant force is zero. But if not, set $p(t+\Delta t)$ as the new coordinates of the predicted mass.

- Step 3: Make a position compensation $\Delta p$ for the predicted coordinates according to the constraint condition, and then eliminate all of the external forces that have been imposed. After that, output the vertex coordinates to Step 4 and turn to Step 2 for further iteration.

- Step 4: Render the garment model according to the updated vertex coordinates.

\section{EXPERIMENTAL SIMULATION RESULT}

This algorithm utilizes Visual C\# and Unity3D to complete the simulation and calculation in Windows 8.1 with the operating environment provided as below: Choose Intel Xeon E5-2620 v2 @2.10GHz as the CPU and NVIDIA Quadro K4000 as the VGA card with 2G of the video memory and 32G of the RAM. With simple computation, this algorithm is able to guarantee a smooth simulation result, showing its advantage in the efficiency, proving that this algorithm is able to realize real-time processing on the influence of the external forces on the cloth. In this experiment, the external forces include gravity and wind power. Figure 5 shows the screenshot of the simulation effect. The left image shows the initial state of the model under the effect of the gravity. The middle image shows the state of the model when the value of the wind power is 1.0 and the right image shows the state when the value of the wind power is 10.0 .
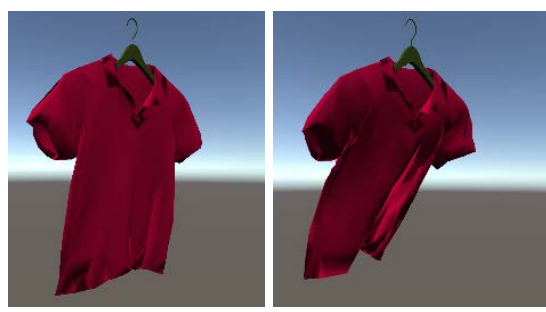

Figure 5. Simulation results of the T-shirt consisting of triangle faces.

In order to verify the performance efficiency of the algorithm, models with different precisions are adopted in this experiment with the specific approach provided as below: perform reduction process on the same garment model based on different precisions to obtain respectively Model 10600, Model 5280 and Model 1028. The more precisely the particle grid is divided, the closer the grid is to the size of the internal discontinuous elements in the cloth, giving a more authentic simulation effect. However undoubtedly, this method will bring about the increase in the computation amount. Actually, with the refinement of the grid division, the computation time has been increased geometrically. Therefore, it would be a direction of efforts in the future to find such a computing method that is quicker with higher precision.

\section{CONCLUSIONS}

With the continuous development of the industrial requirements on garment simulation, it becomes urgent to find such a rapid and universal method that is able to satisfy the requirement of garment simulation in every respect. The problems that must be solved through the algorithm include: I. Satisfaction of the requirements on universality. Since ordinary 3D model is not of regular grid structure, then the vertexes of the triangle face will be mapped into a structural or a flexural spring according to the angular distribution. II. The prediction mechanism with the introduction of vertex position. It guarantees the robustness without the explicit calculation made on the movement speed of the vertex. Also it provides an opportunity for constraint computation and 
position correction. III. The application of rigid spring without any stretch ability to solve effectively the problem of "over-stretching". The algorithm has the advantage in the execution speed, since only vertex position will be calculated in a triangle face, laying a foundation for the real-time garment simulation. Moreover, from the perspective of application, this algorithm is also featured with the characteristics of simplicity and modularization, which are of great importance in the transformation of the practical and maintainable applications and practices.

\section{ACKNOWLEDGEMENT}

This paper is sponsored by Beijing Education Commission scientific research Support Program (KYJH02150201).

\section{REFERENCES}

[1] Provot X. "Deformation constraints in a mass-spring model to describe rigid cloth behavior.” in Proceedings Graphics Interface 95. Quebec: Elsevier. 1995: 147-154.

[2] Baraff D, Witkin A. "Large Steps in Cloth Simulation.” Computer Graphics, 1998, 32(2): 106-117.

[3] Chen Liang, Zhao Shuguang and Zhang Lijuan e.t. "History and research hot spot of three-dimensional virtual garment display." Journal of Textile Research, 2011, 32(10): 153-160.

[4] Ji Feng, Li Ruqin. "Fabric simulation model based-on particle-spring system.” Journal of Textile Research, 2004, 25(1): 42-44.

[5] Wang Xiaobin, Hou Junyi and Shen Xukun. "Application of Interaction Technique in Cloth Animation Based on Physically Modeling.” Journal of System Simulation, 2014, 26(9): 2050-2055.

[6] Liang Xiuxia, Han Huijian and Zhang Caiming. "Survey of cloth animation based on physical simulation." Journal of Computer Research and Development, 2014, 51(1):31-40.

[7] Zhang Dong, Jia Shiyu and Wang Liang. "Particle model with accurate mechanical formulation in cloth simulation." System Simulation Technology, 2008, 4(2):138-142.
[8] Mao Tianlu, Wang Zhaoqi and Xia Shihong. "An algorithm for collision detection and response between human body model and cloth in 3D garment simulation.” Journal of Computer Research and Development, 2006, 43(2): 356-361.

[9] Jiang Yan, Liu Zhengdong, Chen Chunli. "3D textile simulation based on fabric mechanical properties." Journal of Computer-Aided Desigh \& Computer Graphics, 2012, 24(3): 323-329.

[10] Huang Xin, Yang Wenzhen and Wu Xinli e.t. "Virtual fabric haptic rendering with double haptic interactive points." Journal of System Simulation, 2014, 26(9): 1997-2002.

[11] Liu Hui, Chen Chun and Shi Baile. "Simulation of 3D garment based on improved spring-Mass model.” Journal of Software, 2003, 14(3): 619-627.

[12] Chen Qingqing. "Three-Dimensional garment simulation technology based on interactive suture setting." Computer System \& Application, 2013, 22(4): 88-91.

[13] Zhu Guangzhou, Li Xiaojiu. Study on virtual simulation of 2-D patterns [J]. Journal of Textile Research, 2014, 35(10): 141-145.

[14] Vassilev T, Spanlang B. "Efficient cloth model for dressing animated virtual people.” Proc of Learning to Behave Workshop. Enschede the Netherlands:[sn]. 2000: 89-100.

[15] Sun Shouqian, Xu Aiguo and Huang Qi e.t. "Real-time garment simulation based on geometrically approximation character with colliding objects.” Journal of Software, 2007, 18(11): 2921-2931.

[16] Chen Yan, Xue Yuan and Yang Ruoyu. "Real-time simulation for 3D-dressing of random clothes and human body." Journal of Computer Applications, 2014, 34(1): 124-128.

[17] Zhang Yisheng, Cui Shubiao and Liang Shuyun. "Rebuild adjacency topology relation between faces of STL model." Computer Application \& Software, 2001, 18(3): 47-50.

[18] Goldenthal R. "Efficient simulation of inextensible cloth." Acm Transactions on Graphics, 2007, 26(3):49-55. 